\title{
Synthesis of $N$-Alkylated 4-Fluoro-5-phenylpyrrole-2-carboxylate via Isolable Pyrroline Ionic Intermediates
}

\author{
Sung Kwan Kim, Changsoo Jun, Kyungchell Kwak, Kwangyong Park, and Kyuyun Chai ${ }^{\star}$ \\ Department of Bionanochemistry, Wonkwang University, 1ksan, Jeonbuk 570-136, Korea. "E-mail: getyoon@wonkwang.ackkr \\ 'School of Chemical Engineering and Material Science, Chung-Ang University, Seoul 155-756, Korea \\ Received June 28, 2007
}

\begin{abstract}
Organic fluorine chemistry produces many useful products. This paper elucidates the reaction of ethyl-4,4difluoro-2-iodo-5-oxo-5-phenylpentanoate (2) with primary amines in a one-pot scheme. The reaction produced a series of $\beta$-lluoropyrrole derivatives at ambient temperatures. In this reaction, the less bulky the primary amine the higher was the resultant yield. When (2) and aqueous methylamine ( $40 \%$ ) were allowed to react below $0{ }^{\circ} \mathrm{C}$, 5-(ethoxycarboxyl)-1-methyl-3,3-ditluoro-2-hydroxy-2-phenylpyrrolidine, an intermediate molecule for 2-ethyl-4-1louro-1-methyl-5-phenylpyrrole-2-carboxylate (5), was isolated first. Then, (5) reacted with hydroperchloric acid and acetic anhydride to form 5-(ethoxycarboxyl)-1-methyl-3,3-difluoro-2phenylpyrrolinium perchlorate (6). which was converted to 2-ethyl-4-tlouro-1-methyl-5-phenylpyrrole-2carboxylate gradually in the presence of a base. Our experiments demonstrate that the formation of 2-ethyl-4flouro-1-methyl-5-phenylpyrrole-2-carboxylate occurs via both one-pot schemes and stepwise pathways, depending on the reaction conditions. The isolation and characterization of the isolated intermediate (6) suggest an anionic pathway for this reaction.
\end{abstract}

Key Words : $\beta$-Fluoropyrrole, Pyrrole derivatives, Anionic pathway

\section{Introduction}

Organic fluorine chemistry has attracted wide interest in pharmaceutical and medical research involving dnug delivery and physiological activities. ${ }^{1}$ Recent advances in medicine are partially based on the development of proper compounds of biological activities. ${ }^{2}$ Especially, selective fluorination of organic compounds is very important because their biological activities can be greatly modified by the presence of fluorine atoms at specific sites in the molecules. ${ }^{2}$

Pyrrole derivatives have been examined thoroughly for antimicrobial activity because of the recent resurgence of tuberculosis, ${ }^{3}$ which is due to several factors, three of which are the most difficult to overcome. They are multiple drugresistant, or MDR, strains of Mycobacterium tuberculosis versus the conventional therapeutic regimens, ${ }^{3-5}$ the particular vinulence of $\mathrm{M}$. avium, very often responsible for the death of HIV-infected patients, and the particularly resistant mycobacterium cell wall structure, which is very waxy and hydrophobic, with a high lipid content. The cell wall presents a lipidic bilayer and a peptidoglican layer (as that of the bacteria) and a thick mycolate rich outer covering that functions as an exceptionally efficient barrier. Thus, in this case, the search for new active compounds possessing a different mode of action could be very useful. Previous studies on the effect of pyrrole derivatives modified their boundary substituents systematically.

However, pyrroles with fluorine atoms directly attached have not been studied for this purpose yet. Selectively fluorinated pyrrole compounds also have been known to exhibit valuable biological properties, such as fungicidal, bactericidal, and analgesic activities. ${ }^{6}$
Only a few synthetic methods for the preparation of $\beta$ fluoropyrroles have been reported. They include the ring expansion of 2-azid-3,3-difluorocyclobutene, ${ }^{7}$ the thermal $[2+3]$ cycloaddition of 2-carbonethoxy-1-t-butyl-aziridine with chlorotrifluoroethylene, ${ }^{8}$ the photoreaction of pyrrole$\beta$-diazoniumtetrafluoroborate, ${ }^{9}$ and the reaction of 3-bromo1 -(triisopropylsilyl)pyrrole with $\mathrm{N}$-fluorobenzene sulfonamide. ${ }^{10}$ However, all of these methods have drawbacks inherent in their multiple reaction schemes, and relatively harsh reaction conditions would further limit economic use of them. Moreover, these methods often require the blocking of the amino group in the pyrroles before the introduction of substituents to the pyrrole ring.

In the present report, we develop better economic preparative methods via a reduced reaction scheme. We herein report the synthesis of 2-carboxylate derivatives of $\mathrm{N}$-substituted- $\beta$-fluoropyrroles from ethyl-4,4-difluoro-2-iodo-5phenylpentanoate, which works by a very efficient pathway under mild conditions. A possible reaction mechanism for the synthesis is suggested, which is supported by the separation and identification of the intermediates.

\section{Experimental Section}

General. ${ }^{19} \mathrm{~F}$ NMR were recorded on a JEOL FX90Q $(83.81 \mathrm{MHz})$ or Brïker AC-300 (282.44 MHz); spectra were recorded on the AC-300 spectrometer. All samples were taken in $\mathrm{CDCl}_{3}$ solvent and all chemical shifts are reported in parts per million downfield (positive) of the standard: TMS of ${ }^{1} \mathrm{H}$ and ${ }^{13} \mathrm{C} ; \mathrm{CFCl}_{3}$ for ${ }^{19} \mathrm{~F}$ NMR. FT-IR spectra were recorded as $\mathrm{CCl}_{4}$ solutions and reported in wavenumber $\left(\mathrm{cm}^{-1}\right)$ GC-MS spectra were obtained at $70 \mathrm{eV}$ in the 
electron impact mode. High-resolution mass spectral determinations were made at the University of Iowa High Resolution Mass Spectrometry Facility of the Midwest Center for Mass Spectrometry. (Editor's Note: The Midwest Center for Mass Spectrometry is listed as being located at the University of Nebraska in Lincoln.)

5-(Ethoxycarboxyl)-1-methyl-3,3-difluoro-2-hydroxy2-phenylpyrrolidine (5). A $500 \mathrm{~mL}$ round bottom flask was charged with $0.78 \mathrm{~mL}$ of methylamine and $1 \mathrm{~mL}$ water $(40 \%$ $\mathrm{H}_{2} \mathrm{O}$ solution). The solution was cooled at $0^{\circ} \mathrm{C}$; then $0.95 \mathrm{~g}$ (2.5 mmol) of 2 was added via syringe. After 1 hour, the reaction mixture was extracted with $5 \mathrm{~mL}$ dichloromethane, washed in deionized water, and separated by column chromatography $\left(\mathrm{R}_{1}: 0.73\right)$ (ethylacetate $/ n$-hexane, $\left.1: 3\right)$. The white solid of diastereomeric mixture $5(0.65 \mathrm{~g}, 92 \%)$ was obtained. $\mathrm{mp}=85-86{ }^{\circ} \mathrm{C} ;{ }^{19} \mathrm{~F}-\mathrm{NMR}\left(\mathrm{CDCl}_{3}\right) \delta-93.91(\mathrm{~d}, \mathrm{t}$, $\left.J_{\mathrm{TT}}=226.8 \mathrm{~Hz}, J_{\mathrm{IT}}=17.8 \mathrm{~Hz}\right),-98.54\left(\mathrm{~d}, \mathrm{t}, J_{\mathrm{TF}}=228.9 \mathrm{~Hz}\right.$, $\left.J_{\mathrm{ITT}}=21.6 \mathrm{~Hz}\right),-109.10\left(\mathrm{dd}, J_{\mathrm{TF}}=226.3 \mathrm{~Hz}, J_{\mathrm{ITF}}=5.1 \mathrm{~Hz}\right)$, $-115.49\left(\mathrm{dd}, J_{\mathrm{TF}}=226.4 \mathrm{~Hz}, J_{\mathrm{ITF}}=10.2 \mathrm{~Hz}\right.$ ); ${ }^{1} \mathrm{H}-\mathrm{NMR}$ $\left(\mathrm{CDCl}_{3}\right): \delta 7.61(\mathrm{~m}, 2 \mathrm{H}), 7.36(\mathrm{~m}, 3 \mathrm{H}), 4.83(-\mathrm{OH}), 4.24(\mathrm{~m}$, $2 \mathrm{H}), 4.13$ and $3.76(\mathrm{~m}, 1 \mathrm{H}), 2.77(\mathrm{~m}, 1 \mathrm{H}), 2.50(\mathrm{~m}, 1 \mathrm{H}), 2.35$ and $2.30\left(-\mathrm{NCH}_{3}\right), 1.30(\mathrm{t}, J=7.2 \mathrm{~Hz}) ;{ }^{13} \mathrm{C} \mathrm{NMR}\left(\mathrm{CDCl}_{3}\right): \delta$ $179.14(\mathrm{~s}), 172.10(\mathrm{~s}), 135.77(\mathrm{~s}), 135.56(\mathrm{~s}), 128.77(\mathrm{~s})$, $128.32(\mathrm{~s}), 128.24(\mathrm{~s}), 137.98(\mathrm{~s}), 127.70(\mathrm{~s}), 125.15\left(\mathrm{dd}, J_{\mathrm{FF}}\right.$ $\left.=63.5 \mathrm{~Hz}, J_{\mathrm{FH}}=3.5 \mathrm{~Hz}\right), 121.74\left(\mathrm{~d}, J_{\mathrm{FT}}=61.2 \mathrm{~Hz}\right), 93.80(\mathrm{t}$, $J=25.7 \mathrm{~Hz}), 92.11(\mathrm{t}, J=25.0 \mathrm{~Hz}), 68.27(\mathrm{~s}), 61.15(\mathrm{~s})$, $60.88(\mathrm{~s}), 36.34(\mathrm{t}, J=26.9 \mathrm{~Hz}), 35.06(\mathrm{t}, J=25.3 \mathrm{~Hz}), 32.41$ (s), 29.89 (s) $14.10(\mathrm{~s}), 13.99$ (s); FT-IR $\left(\mathrm{CCl}_{4}\right) 3533,2996$, $1653\left(\mathrm{~cm}^{-1}\right)$; GC-MS (relative intensity) $285\left(\mathrm{M}^{+}\right), 268(-\mathrm{OH}$, $30.01), 266\left(-\mathrm{C}_{2} \mathrm{H}_{4}, 37.05\right)$. HRMS observed, 285.1125, $\mathrm{C}_{14} \mathrm{H}_{17} \mathrm{NO}_{3} \mathrm{~F}_{2}$, calculated, 285.1177 .

5-(Ethoxycarboxyl)-1-methyl-3,3-difluoro-2-phenylpyrrolinium perchlorate $(6)$. A $50 \mathrm{~mL}$ round flask was charged with $0.5 \mathrm{~g}(1.8 \mathrm{mmol})$ of 5 and $1.3 \mathrm{~mL}$ of acetic anhydride, and $1.6 \mathrm{~mL}(2.7 \mathrm{mmol})$ of hydroperchloric acid was added under nitrogen gas. After the flask was stirred for 20 minutes, anhydrous diethyl ether $(5 \mathrm{~mL})$ was added. The colorless compound was crystallized out and filtered before re-crystallization from dried dichloromethane and anhydrous ethyl ether. A white solid $6(0.59 \mathrm{~g}, 91 \%)$ was obtained. mp $140-142{ }^{\circ} \mathrm{C}$; ${ }^{19} \mathrm{~F}$ NMR (DMSO-d $\mathrm{d}_{6}$ and $\mathrm{CDCl}_{3}$ ) -95.38 (ddd, $J=288.9 \mathrm{~Hz}, 21.7 \mathrm{~Hz}, 9.03 \mathrm{~Hz}, 1 \mathrm{~F}$ ), -93.88 (dddd, $J=$ $267.3 \mathrm{~Hz}, 19.9 \mathrm{~Hz}, 9.0 \mathrm{~Hz}, 9.0 \mathrm{~Hz}, 1 \mathrm{~F}$ ); 'H-NMR (DMSO-d 6 and $\left.\mathrm{CDCl}_{3}\right) \delta 7.80(\mathrm{~m}, 5 \mathrm{H}), 4.40(\mathrm{t}, J=7.0 \mathrm{~Hz}), 4.02(\mathrm{~s}, 1 \mathrm{H})$, $3.15(\mathrm{~m}, 3 \mathrm{H}), 1.38(\mathrm{t}, J=7.2 \mathrm{~Hz}) ;{ }^{13} \mathrm{C} \mathrm{NMR}$ (DMSO-d $\mathrm{d}_{6}$ and $\left.\mathrm{CDCl}_{3}\right) \& 174.07(\mathrm{t}, J=29.9 \mathrm{~Hz}), 164.48(\mathrm{~s}), 136.15(\mathrm{~s})$, $130.13(\mathrm{~s}), 129.27(\mathrm{~s}), 128.27(\mathrm{~s}), 125.50(\mathrm{~d}, J=250.3 \mathrm{~Hz})$, $122.15(\mathrm{~d}, J=250.3 \mathrm{~Hz}), 120.86(\mathrm{~s}), 69.85(\mathrm{~s}), 63.40(\mathrm{~s})$, $41.05(\mathrm{~s}), 34.25$ (t, $J=24.0 \mathrm{~Hz}), 13.10(\mathrm{~s})$; FT-IR $\left(\mathrm{CCL}_{4}\right)$ $2998,2338,2310,1717,1616,1406\left(\mathrm{~cm}^{-1}\right)$. HRMS observed, 332.0854, $\mathrm{C}_{14} \mathrm{H}_{16} \mathrm{NO}_{6} \mathrm{~F}_{2}$, calculated, 332.0946.

Ethyl-4-fluoro-1H-5-phenylpyrrole-2-carboxylate (4a). A $50 \mathrm{~mL}$ round flask was charged with $0.3 \mathrm{~mL}$ aqueous ammonia solution $\left(29 \% \mathrm{H}_{2} \mathrm{O}\right.$ solution) and $1 \mathrm{~mL}$ water. The solution was cooled at $0{ }^{\circ} \mathrm{C}$ and then $0.5 \mathrm{~g}(1.3 \mathrm{mmol})$ starting material (2) was added slowly via a syringe. After 1 hour of stirring, the reaction mixture was extracted with 10
$\mathrm{mL}$ dichloromethane, washed with water, dried with anhydrous magnesium sulfate, filtered and evaporated. The residue was purified by column chromatography $\left(\mathrm{R}_{\mathrm{j}}: 0.68\right.$ ) (ethyl acetate $/ n$-hexane, $1: 3)$. A white solid $4 \mathrm{a}(0.22 \mathrm{~g}, 73 \%)$ was obtained, mp 110-111 ${ }^{\circ} \mathrm{C} ;{ }^{19} \mathrm{~F} \mathrm{NMR}\left(\mathrm{CDCl}_{3}\right) \delta-159.25$ (s, $1 \mathrm{~F}) ;{ }^{1} \mathrm{H} \mathrm{NMR}\left(\mathrm{CDCl}_{3}\right) \delta 7.59(\mathrm{~d}, J=7.5 \mathrm{~Hz}, 2 \mathrm{H}), 7.29(\mathrm{t}, J=$ $7.6 \mathrm{~Hz}, 2 \mathrm{H}), 7.18(\mathrm{t}, J=7.4 \mathrm{~Hz}, 1 \mathrm{H}), 6.59(\mathrm{~d}, J=2.8 \mathrm{~Hz}$, $1 \mathrm{H}), 4.20(\mathfrak{q}, J=7.3 \mathrm{~Hz}, 2 \mathrm{H}), 1.23(\mathrm{t}, J=7.1 \mathrm{~Hz}, 3 \mathrm{H}) ;{ }^{13} \mathrm{C}$ NMR $\left(\mathrm{CDCl}_{3}\right) \delta 129.13(\mathrm{~d}, J=4.9 \mathrm{~Hz}), 128.77(\mathrm{~s}, J=127.5$ $\mathrm{Hz}), 125.25$ (d, $J=4.5 \mathrm{~Hz}), 161.43$ (d, $J=3.6 \mathrm{~Hz}), 120.93$ $(\mathrm{d}, J=19.1 \mathrm{~Hz}), 117.86(\mathrm{~d}, J=7.3 \mathrm{~Hz}), 103.34$ (d, $J=15.6$ $\mathrm{Hz}), 60.84(\mathrm{~s}), 14.30$ (s); FT-IR $\left(\mathrm{CCl}_{4}\right) 2959,1635\left(\mathrm{~cm}^{-1}\right)$; GC-MS (relative intensity) $233\left(\mathrm{M}^{+}\right), 187(-\mathrm{OEt}, 100)$; HRMS observed, $233.0826\left(\mathrm{M}^{+}\right), \mathrm{C}_{13} \mathrm{H}_{12} \mathrm{O}_{2} \mathrm{NF}$, calculated, 233.0852,

Ethyl-4-fluoro-1-methyl-5-phenylpyrrole-2-carboxylate (4b). (a) A $50 \mathrm{~mL}$ round flask was charged with $0.27 \mathrm{~g}$ (1 mmol) of 5 and acetic anhydride $(1.3 \mathrm{~mL})$ and the reaction mixture was stirred magnetically for 20 minutes at room temperature, followed by addition of $5 \mathrm{~mL}$ of dichloromethane and $0.7 \mathrm{~mL}(5 \mathrm{mmol})$ of triethyl amine. After this solution was stirred for 3 hours, $5 \mathrm{~mL}$ of $0.01 \mathrm{~N}-\mathrm{HCl}$ was added, followed by extraction with $5 \mathrm{~mL}$ dichloromethane, washing with saturated $\mathrm{NaHCO}_{3}$ and brine, drying and evaporation. The yield of liquid was $97 \%(0.25 \mathrm{~g}) .99 .9 \%$ GC purity. (b) $0.068 \mathrm{~g}(1 \mathrm{mmol})$ of 5 and $0.56 \mathrm{~mL}$ triethyl amine were placed in $5 \mathrm{~mL}$ dichloromethane. After the solution was stirred for $3 \mathrm{hr}$ at room temperature, it was worked up with a similar procedure (a) to give liquid, 7 $(0.25 \mathrm{~g}, 99 \%) .{ }^{19} \mathrm{~F}$ NMR $\left(\mathrm{CDCl}_{3}\right) \delta-165.53$ (s); 'H NMR $\left(\mathrm{CDCl}_{3}\right) \delta 7.25-7.40(\mathrm{~m}, 5 \mathrm{H}), 6.65(\mathrm{~s}, 1 \mathrm{H}), 4.108(\mathrm{q}, J=7.1$ $\mathrm{Hz}, 2 \mathrm{H}), 3.75(\mathrm{~s}, 3 \mathrm{H}), 1.25(\mathrm{t}, J=7.1 \mathrm{~Hz}, 3 \mathrm{H}) ;{ }^{13} \mathrm{C} \mathrm{NMR}$ $\left(\mathrm{CDCl}_{3}\right) \delta 162.02(\mathrm{~s}), 147.53(\mathrm{~d}, J=241.7 \mathrm{~Hz}), 125.32(\mathrm{~d}, J$ $=22.0 \mathrm{~Hz}), 118.58(\mathrm{~d}, J=6.3 \mathrm{~Hz}), 103.41(\mathrm{~d}, J=14.9 \mathrm{~Hz})$, 59.95 (s), 34.12 (s), 14.39 (s); FT-IR $\left(\mathrm{CCl}_{4}\right) 3026,1607$, $1581\left(\mathrm{~cm}^{-1}\right)$; GC-MS (relative intensity) $247\left(\mathrm{M}^{+}, 53.15\right)$, 248 (7.87), 219 (-C2H4, 35.49), 202 (-OEt, 35.31), 175 (53.45), 133 (100); HRMS observed, 247.0978, $\mathrm{C}_{14} \mathrm{H}_{14} \mathrm{NO}_{2} \mathrm{~F}$, calculated, 247.1009 .

Ethyl-4-fluoro-1-ethyl-5-phenylpyrrole-2-carboxylate (4c). Using the same method as in $(\mathbf{4 b})$, the reaction of ethyl 4,4-difluoro-2-iodo-5-oxo-5-phenylpentanoate $(0.4 \mathrm{~g}, 1$ mol) and ethylamine $(0.26 \mathrm{~g}, 4 \mathrm{mmol}, 70 \%$ aqueous solution) produced ethyl-4-fluoro-1-ethyl-5-phenylpyrrole-2carboxylate $(0.25 \mathrm{~g}, 96 \%)$.

'H NMR $\left(\mathrm{CDCl}_{3}\right): \delta 7.46-7.37(\mathrm{~m}, 5 \mathrm{H}), 6.72(\mathrm{~s}, 1 \mathrm{H}), 4.31$ $4.25(\mathrm{~m}, 4 \mathrm{H}), 1.34(\mathrm{t}, J=7.3 \mathrm{~Hz}, 3 \mathrm{H}), 1.32(\mathrm{t}, J=7.3 \mathrm{~Hz}$, 3H). ${ }^{19} \mathrm{~F}$ NMR ( $\left.\mathrm{CDCl}_{3}, \mathrm{CFCl}_{3}\right): \delta-165.62(\mathrm{~s}) .{ }^{13} \mathrm{C} \mathrm{NMR}$ $\left(\mathrm{CDCl}_{3}\right): \delta 160.77(\mathrm{~d}, J=2.9 \mathrm{~Hz}), 147.76(\mathrm{~d}, J=241.8 \mathrm{~Hz})$, 130.14 (s), 128.73 (s), 128.62 (s), $128.46(\mathrm{~d}, J=2.9 \mathrm{~Hz}$ ), 124.93 (d, $J=22.0 \mathrm{~Hz}), 117.40$ (d, $J=6.7 \mathrm{~Hz}), 104.07$ (d, $J$ $=15.4 \mathrm{~Hz}), 60.08(\mathrm{~s}), 40.93(\mathrm{~s}), 16.95(\mathrm{~s}), 14.45(\mathrm{~s}) . \mathrm{IR}$ $\left(\mathrm{CDCl}_{3}\right): 2984,1701,1566,1458,1421 \mathrm{~cm}^{-1}$. GC-MS (m/e, relative intensity); $261\left(\mathrm{M}^{+}, 96.96\right), 216(29.38), 188$ (46.94), 158 (24.93), 133 (31.04). HRMS observed, 261.1113, $\mathrm{C}_{15} \mathrm{H}_{16} \mathrm{NO}_{2} \mathrm{~F}$, calculated, 261.1165.

Ethyl-4-fluoro-1-propyl-5-phenylpyrrole-2-carboxylate 
(4d). Using the method in (4b), ethyl-4-fluoro-1-propyl-5phenylpyrrole-2-carboxylate $(0.25 \mathrm{~g}, 85 \%)$ was formed from the reaction of ethyl 4,4-difluoro-2-iodo-5-oxo-5-phenylpentanoate $(0.4 \mathrm{~g}, 1 \mathrm{mmol})$ and propylamine $(0.24 \mathrm{~g}, 4$ mmol).

${ }^{1} \mathrm{H} \mathrm{NMR}\left(\mathrm{CDCl}_{3}\right): \delta 7.48-7.37(\mathrm{~m}, 5 \mathrm{H}), 6.74(\mathrm{~s}, 1 \mathrm{H}), 4.29$ (q, $J=7.3 \mathrm{~Hz}, 2 \mathrm{H}), 4.23$ (t, $J=7.3 \mathrm{~Hz}$ ), 1.62 (sextet, $J=7.3$ $\mathrm{Hz}, 2 \mathrm{H}), 1.35(\mathrm{t}, J=7.3 \mathrm{~Hz}, 3 \mathrm{H}), 0.71(\mathrm{t}, J=7.3 \mathrm{~Hz}, 3 \mathrm{H}) .{ }^{19} \mathrm{~F}$ $\mathrm{NMR}\left(\mathrm{CDCl}_{3}, \mathrm{CFCl}_{3}\right): \delta-165.86(\mathrm{~S}) .{ }^{13} \mathrm{C} \mathrm{NMR}\left(\mathrm{CDCl}_{3}\right): \delta$ $161.08(\mathrm{~d}, J=2.9 \mathrm{~Hz}), 147.90(\mathrm{~d}, J=241.8 \mathrm{~Hz}) .130 .32(\mathrm{~s})$, $128.89(\mathrm{~s}), 128.75(\mathrm{~d}, J=3.8 \mathrm{~Hz}$,) $128.71(\mathrm{~s}) 125.58(\mathrm{~d}, J=$ $22.1 \mathrm{~Hz}) 117.99(\mathrm{~d}, J=6.7 \mathrm{~Hz}), 104.30(\mathrm{~d}, J=15.4 \mathrm{~Hz})$, $60.25(\mathrm{~s}), 47.53(\mathrm{~s}), 25.11(\mathrm{~s}), 14.63(\mathrm{~s}), 11.08(\mathrm{~s}) . \mathrm{R}\left(\mathrm{CCl}_{4}\right)$ : $3020,2990,1708,1566,1458,1420,1261,1213 \mathrm{~cm}^{-1}$. GCMS (m/e, relative intensity): $275\left(\mathrm{M}^{+}, 100\right), 246(7.99), 230$ (26.68), 202 (71.72), 174 (45.33), 133 (149.40), 57 (11.60). HRMS observed, $275.1209, \mathrm{C}_{16} \mathrm{H}_{18} \mathrm{NO}_{2} \mathrm{~F}$, calculated 275.1322 .

Ethyl-4-fluoro-1-butyl-5-phenylpyrrole-2-carboxylate (4e). Following the method (4b), ethyl-4-fluoro-1-butyl-5phenylpyrrole-2-carboxylate $(0.23 \mathrm{~g}, 0.86 \mathrm{mmol}, 88 \%)$ was formed from the reaction of ethyl 4,4-difluoro-2-iodo-5-oxo5 -phenylpentanoate $(0.4 \mathrm{~g}, 1 \mathrm{mmol})$ and butylamine $(0.29 \mathrm{~g}$, $4 \mathrm{mmol}$ ).

${ }^{1} \mathrm{H}$ NMR $\left(\mathrm{CDCl}_{3}\right): \delta 7.47-7.44(\mathrm{~m}, 2 \mathrm{H}), 7.41-7.37(\mathrm{~m}$, $3 \mathrm{H}), 6.73(\mathrm{~s}, 1 \mathrm{H}) 4.28(\mathrm{q}, J=7.3 \mathrm{~Hz}), 4.26(\mathrm{q}, J=7.3 \mathrm{~Hz}$, $2 \mathrm{H}), 1.55(\mathrm{~m}, 2 \mathrm{H}) 1.34(\mathrm{t}, J=7.3 \mathrm{~Hz}, 3 \mathrm{H}), 1.13(\mathrm{~m}, 2 \mathrm{H})$, $0.75(\mathrm{t}, J=7.3 \mathrm{~Hz}, 3 \mathrm{H}) .{ }^{10} \mathrm{~F}$ NMR $\left(\mathrm{CDCl}_{3}, \mathrm{CFCl} 3\right): \delta$ $-165.83(\mathrm{~s}) .{ }^{13} \mathrm{C}$ NMR $\left(\mathrm{CDCl}_{3}\right): \delta 161.04(\mathrm{~d}, J=2.9 \mathrm{~Hz})$, $147.89(\mathrm{~d}, J=241.8 \mathrm{~Hz}), 130.32(\mathrm{~s}), 128.86(\mathrm{~s}), 128.70(\mathrm{~s})$, 128.56 (s), 125.52 (d, $J=22.1 \mathrm{~Hz}), 117.93$ (d, $J=6.7 \mathrm{~Hz}$ ), $104.29(\mathrm{~d}, J=15.4 \mathrm{~Hz}), 60.23(\mathrm{~s}), 45.82(\mathrm{~s}), 33.89(\mathrm{~s}), 19.84$ (s), 14.62 (s), 13.75 (s). IR ( $\left.\mathrm{CDCl}_{3}\right)$ : 3030, 2985, 2922, $1701,1567,1457,1248,1214 \mathrm{~cm}^{-1}$. GC-MS (m/e, relative intensity): 290 (1.049), 289 (62.05), 247 (28.36), 246 $(35.91), 244(13.80), 218(10.24), 217(13.48), 216(69.12)$, 187 (44.47), 175 (19.84), 174 (100), $158(25.22), 133$ (35.58), 59 (11.37), 55 (15.95). HRMS observed, 289.1315, $\mathrm{C}_{17} \mathrm{H}_{20} \mathrm{NO}_{2} \mathrm{~F}$, calculated, 289.1478 .

Ethyl-4-fluoro-1-cyclopentyl-5-phenylpyrrole-2-carboxylate (4f). ethyl-4-fluoro-1-cyclopentyl-5-phenylpyrrole-2carboxylate $(0.22 \mathrm{~g}, 0.73 \mathrm{mmol}, 88 \%)$ was formed from the reaction of ethyl 4,4-difluoro-2-iodo-5-oxo-5-phenylpentanoate $(0.4 \mathrm{~g}, 1 \mathrm{mmol})$ and cyclopentylamine $(0.35 \mathrm{~g}, 4$ mmol).

${ }^{1} \mathrm{H} \mathrm{NMR}\left(\mathrm{CDCl}_{3}\right): \delta 7.48-7.37(\mathrm{~m}, 5 \mathrm{H}), 6.81(\mathrm{~s} 1 \mathrm{H}), 4.68$ (quintet, $J=9.2 \mathrm{~Hz}, 1 \mathrm{H}), 4.27(\mathrm{q}, J=7.3 \mathrm{~Hz}, 2 \mathrm{H}), 2.33(\mathrm{~m}$, $2 \mathrm{H}), 1.88(\mathrm{~m}, 2 \mathrm{H}), 1.60(\mathrm{~m}, 2 \mathrm{H}), 1.49(\mathrm{~m}, 2 \mathrm{H}), 1.35(\mathrm{t}, J=$ $7.3 \mathrm{~Hz}, 3 \mathrm{H}) \cdot{ }^{19} \mathrm{~F} \mathrm{NMR}\left(\mathrm{CDCl}_{3}, \mathrm{CFCl}_{3}\right): \delta-165.84(\mathrm{~s}) \cdot{ }^{13} \mathrm{C}$ NMR $\left(\mathrm{CDCl}_{3}\right): \delta 160.92(\mathrm{~d}, J=2.9 \mathrm{~Hz}), 147.54(\mathrm{~d}, J=$ $240.9 \mathrm{~Hz}), 130.59$ (s), 129.67 (d, $J=2.9 \mathrm{~Hz}), 128.65$ (s), 128.45 (s), 126.60 (d, $J=22.1 \mathrm{~Hz}), 118.38$ (d, $J=6.7 \mathrm{~Hz}$ ), $105.92(\mathrm{~d}, J=14.4 \mathrm{~Hz}), 60.30(\mathrm{~s}), 59.68(\mathrm{~s}), 31.99(\mathrm{~s}), 34.88$ (s), 14.66 (s). IR ( $\left.\mathrm{CDCl}_{3}\right): 3020,2932,1701,1452,1212$ $\mathrm{cm}^{-1}$. GC-MS (m/e, relative intensity); $301\left(\mathrm{M}^{+}, 19.08\right), 256$ (2.73), $233(58.86), 187(100), 159(21.46), 133(16.30), 104$ (2.36), 67 (12.77), 57 (3.83). HRMS observed, 301.1293, $\mathrm{C}_{18} \mathrm{H}_{20} \mathrm{NO}_{2} \mathrm{~F}$, calculated, 301.1478 .
Ethyl-4-fluoro-1-cyclohexyl-5-phenylpyrrole-2-carboxylate $(\mathbf{4 g})$. ethyl-4-fluoro-1-cyclohexyl-5-phenylpyrrole-2carboxylate $(0.14 \mathrm{~g}, 0.44 \mathrm{mmol}, 44 \%)$ was formed from the reaction of ethyl 4,4-difluoro-2-iodo-5-oxo-5-phenylpentanoate $(0.40 \mathrm{~g}, 1 \mathrm{mmol})$ and cyclohexylamine $(0.40 \mathrm{~g}, 4$ mmol).

'H NMR $\left(\mathrm{CDCl}_{3}\right): \delta 7.47-7.35(\mathrm{~m}, 5 \mathrm{H}), 6.79(\mathrm{~s}), 4.42(\mathrm{~m}$, $1 \mathrm{H}), 4.28(\mathrm{q}, J=7.3 \mathrm{~Hz}, 2 \mathrm{H}), 1.36(\mathrm{t}, J=7.3 \mathrm{~Hz}, 3 \mathrm{H}), 1.28-$ $1.11(\mathrm{~m}, 10 \mathrm{H}) .{ }^{19} \mathrm{~F} \mathrm{NMR}\left(\mathrm{CDCl}_{3}, \mathrm{CFCl}_{3}\right): \delta-125.98(\mathrm{~s}),{ }^{13} \mathrm{C}$ NMR $\left(\mathrm{CDCl}_{3}\right): \delta 160.76(\mathrm{~d}, J=2.9 \mathrm{~Hz}), 147.53(\mathrm{~d}, J=$ $240.9 \mathrm{~Hz}), 130.58(\mathrm{~s}), 129.67(\mathrm{~d}, J=2.9 \mathrm{~Hz}), 128.65(\mathrm{~s})$, $128.45(\mathrm{~s}), 125.87$ (d, $J=22.1 \mathrm{~Hz}), 118.29$ (d, $J=6.7 \mathrm{~Hz}$ ), $105.30(\mathrm{~d}, J=14.4 \mathrm{~Hz}), 60.11(\mathrm{~s}), 58.97(\mathrm{~s}), 29.71(\mathrm{~s}), 26.32$ (s), $24.95(\mathrm{~s}), 14.44(\mathrm{~s})$. IR $\left(\mathrm{CCl}_{4}\right): 3030,2990,2945,1702$, $1606,1552,1456,1421,1296,1213 \mathrm{~cm}^{-1}$. GC-MS (m/e, relative intensity): $315\left(\mathrm{M}^{+}, 65.72\right), 270(4.85), 242(3.30)$, 233 (100), 205 (4.74), 187 (96.00), 159 (11.21), 133 (9.87), 56 (3.72). HRMS observed, 315.1473, $\mathrm{C}_{19} \mathrm{H}_{22} \mathrm{NO}_{2} \mathrm{~F}$, calculated, 315.1635.

Ethyl-4-fluoro-l-benzyl-5-phenylpyrrole-2-carboxylate (4h). ethyl-4-fluoro-1-benzyl-5-phenylpyrrole-2-carboxylate $(0.26 \mathrm{~g}, 0.8 \mathrm{mmol}, 80 \%)$ was formed from the reaction of ethyl 4,4-difluoro-2-iodo-5-oxo-5-phenylpentanoate $(0.4 \mathrm{~g}$, $1 \mathrm{mmol})$ and benzylamine $(0.48 \mathrm{~g}, 4 \mathrm{mmol})$.

'H NMR $\left(\mathrm{CDCl}_{3}\right): \delta 7.29-7.16(\mathrm{~m}, 10 \mathrm{H}), 6.87(\mathrm{~s}, 1 \mathrm{H})$, $5.48(\mathrm{~s}, 2 \mathrm{H}), 4.13(\mathrm{q}, J=7.2 \mathrm{~Hz}, 2 \mathrm{H}), 1.19(\mathrm{t}, J=7.1 \mathrm{~Hz}$, 3H). ${ }^{19} \mathrm{~F}$ NMR ( $\left.\mathrm{CDCl}_{3}, \mathrm{CFCl}_{3}\right): \delta-164.87(\mathrm{~s}) .{ }^{13} \mathrm{C} \mathrm{NMR}$ $\left(\mathrm{CDCl}_{3}\right): \delta 160.83$ (d, $\left.J=2.9 \mathrm{~Hz}\right), 148.14(\mathrm{~d}, J=242.8 \mathrm{~Hz}$ ), $139.07(\mathrm{~s}), 128.88(\mathrm{~s}), 128.70(\mathrm{~s}), 128,24(\mathrm{~d}, J=2.9 \mathrm{~Hz}$ ), $127.18(\mathrm{~s}), 126.01(\mathrm{~d}, J=22.1 \mathrm{~Hz}), 125.87(\mathrm{~s}), 118.72$ (d, $J=$ $6.7 \mathrm{~Hz}), 104.94(\mathrm{~d}, J=15.4 \mathrm{~Hz}), 60.37(\mathrm{~s}), 49.28(\mathrm{~s}), 14.49$ (s) IR $\left(\mathrm{CCl}_{4}\right): 3253,3020,17101,1568,1444,1253,1215$ $\mathrm{cm}^{-1}$. GC-MS (m/e, relative intensity): $323\left(\mathrm{M}^{+}, 81.64\right), 278$ (9.32), $250(10.21), 248(8.81), 92$ (10.81), 91 (100). HRMS observed, 311.1264, $\mathrm{C}_{19} \mathrm{H}_{18} \mathrm{NO}_{2} \mathrm{~F}$, calculated, 311.1322.

\section{Results and Discussion}

Unsubstituted pyrrole is electron rich compared with benzene, with a ratio of pi electron distribution per nuclei of 1.161. The attack of electrophiles in pyrrole is, in general, more facile than in benzene and pyridine. The introduction of fluorine atoms, however, changes the pi electron density of pyrrole, aggravating its electron-rich nature. The synthesis of $\beta$-fluoropyrrole derivatives via difluoro-pyrroles cannot be further enhanced by a mechanism of electrophilic substitution or abstractions on the fluorinated pyrrole rings. Thus, the ring formation reaction of Qui-Burton's and our method $\mathrm{d}^{1-1+}$ in which precursors contain pre-introduced substituents can be a unique choice to prepare selectively substituted $\beta$-fluoropyrrole derivatives with high yields, though the reaction mechanism has not been studied in detail. In the present study, various substituents are introduced on nitrogen to elucidate the nature of this reaction, as shown in Scheme 1.

Scheme 1 shows the synthetic routes of the two N-methyl$\beta$-fluoropyrrole derivatives, ethyl-4-fluoro-5-phenylpyrrole- 
<smiles></smiles>

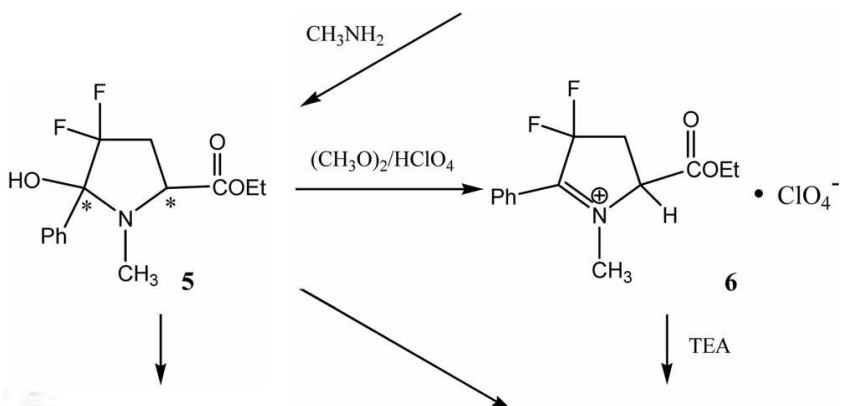<smiles>CCOC(=O)C1CC(F)(F)C(OC)(c2ccccc2)N1C</smiles>

Scheme 1

2-carboxylate (4a) and ethyl-4-fluoro-1-methyl-5-pheny]pyrrol-2-carboxylate (4b), from ethyl-4,4-difluoro-2-iodo-4bezoylate (2), which can be prepared from $\alpha$-chloro- $\alpha, \alpha$ difluoroacetic acid (1), ${ }^{11,13}$ The formation of $4 \mathbf{a}$ is reported as occurring by the reaction of 2 with excess ammonium hydroxide $(29 \%)$ for $24 \mathrm{hr}$ at room temperature. ${ }^{12}$ In our previous work, ${ }^{1+}$ 4b was also synthesized from 2 in high yield under the same reaction conditions. However, when 2 and aqueous methylamine $(40 \%)$ were allowed to react below $0{ }^{\circ} \mathrm{C}$, 5-(ethoxycarboxyl)-1-methyl-3,3-difluoro-2hydroxy-2-phenylpyrrolidine (5), an intermediate molecule for 2-ethyl-4-flouro-1-methyl-5-phenylpyrrole-2-carboxylate $(\mathbf{4 b})$, was isolated first. Then, 5 reacted with hydroperchloric acid and acetic anhydride to form 5-(ethoxycarboxyl)-1-methyl-3,3-difluoro-2-phenylpyrrolinium perchlorate (6), which was converted to 2-ethyl-4-flouro-I-methyl-5phenylpyrrole-2-carboxylate gradually in the presence of triethylamine. Characterization of the isolated intermediate molecules $(5,6)$ revealed unambiguously the involvement of deprotonation and defluorination, namely an anionic pathway, in the course of preparing the targeted $B$-monofluorj- nated pyrroles. In addition, it is noteworthy that the selection of solvent combination to control the basicity of reactants was also crucial to isolate those intermediate, otherwise the reaction is a one-pot process.

When 5 was prepared from 2, 5 mole-times of $40 \%$ aqueous methylamine was cooled to below $0^{\circ} \mathrm{C}$, and then the reaction mixture was diluted 4-5 times with ammonium hydroxide before the reaction proceeded for one-hour. Through this reaction, 5 was obtained in large amount instead of the expected $4 \mathrm{~b}$; the ratio of 5 to $4 \mathrm{~b}$ was measured as 98 to 2 by the interpretation of ${ }^{19} \mathrm{~F}-\mathrm{NMR}$ spectrum of the reaction mixture. The compound $\mathbf{5}$ was isolated by flash chromatography on a silica gel with $1: 3$ ethyl acetate/nhexane as an eluent. The isolated compound 4 exists as a diastereomeric mixture, as shown in the ${ }^{19} \mathrm{~F}-\mathrm{NMR}$ and ${ }^{1} \mathrm{H}-$ NMR spectra in $\mathrm{CDCl}_{3}$. After several days at room temperature, the color of the solution of $5 \mathrm{in} \mathrm{CDCl}_{3}$ changed from pale yellow to red, indicating conversion of 5 into $4 \mathrm{~b}$ spontaneously. The yield was no more than $40 \%$. By replacing methylamine with ammonjum hydroxide under the same reaction conditions, $4 \mathrm{~b}$ was formed from 2 in $73 \%$ yie]d. In this case, however, we failed to isolate the intermediate like 5 , which implies that the conversion from the possible intermediate to 4 is fast in non-aqueous media. Based on these facts, we propose a reaction mechanism as shown in Scheme 2 . In the case of the reaction using aqueous methylamine, the conversion from 5 into the positively charged intermediate (8) is expected to be much slower than the similar conversion process in the reaction using neat ammonium hydroxide. In this case, the resulting transient product by the conversion process is expected to be uncharged.

Furthermore, the intermediate 5 was successfully converted to 6 in high yield. In this reaction, 6 was prepared by the reaction of $\mathbf{5}$ with acetic anhydride and hydroperchloric acid, which is illustrated as the intermediate $(\mathbf{8})$ in Scheme 2. Treatment of 6 with triethylamine in methylene chloride gave 4 in $98 \%$ of yield. In a much simpler way, the final product 4 can also be obtained through the reaction of 5 with acetic anhydride and triethylamine in one step, though 6 may be stored. It is apparent that the current method utilizes a route of anionic pathway. That is, the intermediate molecule (6) has a structure in which $\mathrm{C} 2-\mathrm{H}$ is the most acidic and at which a base attack is expected to happen first. The environment around the $\mathrm{C} 2-\mathrm{H}$ is important to figure out the mechanism of the formation of $\mathbf{4}$ from 6 via a base attack.

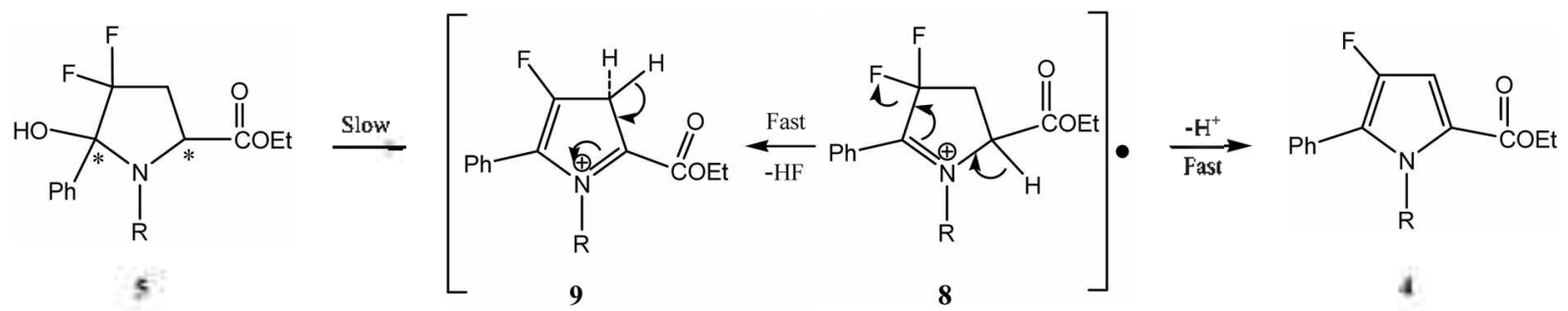

$\mathrm{R}=\mathrm{H}(\mathbf{4 a}), \mathrm{CH}_{3}(\mathbf{4 b}), \mathrm{C}_{2} \mathrm{H}_{5}(\mathbf{4 c})$, cyclopropyl$(\mathbf{4 d})$, butyl(4e), cyclopentyl(4f), cyclohexyl(4g), benzyl(4h) 
The carbonyl ester group, which is an electron withdrawing group, remained on the pyrrole ring, but made the protonic hydrogen vulnerable for a base attack. The reaction of (6) with Lewis bases resulted in the acidic protons leaving in sequence, whereas an electron-rich group, instead of the carbonyl ester, would be expected to leave first. Then, the deprotonation augments the anionic charge followed by removal of one $\beta$-fluorine atom. Therefore we expect that $\beta$ monofluoro-substituted $1 H$-pyrroles compound would form in high yield as well, which contrasts with the results reported previously. ${ }^{15}$ Finally, the reaction of ethyl-4,4-difluoro-2iodo-5-oxo-5-phenylpentanoate (2) with primary amines was controlled within a one-pot scheme, but with aqueous solvents, a series of $\beta$-monofluoropyrrole derivatives was prepared with high yields at ambient temperatures. The yield of products varied only with the bulkness of $N$-substituents. That is, cyclohexylamine gave a yield of $44 \%$, whereas the benzylamine yield was $80 \%$.

The two new compounds 5 and 6 were identified or characterized by ${ }^{19} \mathrm{~F}$ NMR, 'H-NMR, ${ }^{13} \mathrm{C}-\mathrm{NMR}, \mathrm{IR}$, and Mass Spectroscopic data. ${ }^{19} \mathrm{~F}$ NMR data of 6 revealed a typical coupling pattern due to two fluorine atoms. From the Mass and ${ }^{1} \mathrm{H}-\mathrm{NMR}$ data, the structure of 6 could be postulated as 5-(ethoxycarboxyl)-1-methyl-3,3-difluoro-2phenylpyrrolinium perchlorate. The $\mathbb{R}$ data confirms a carbonyl and $\mathrm{C}-\mathrm{H}$ stretches as well as the $\mathrm{C}=\mathrm{N}$ stretch appeared at $1717,2998,1617 \mathrm{~cm}^{-1}$, respectively. ${ }^{1} \mathrm{H}-\mathrm{NMR}$ data taken at room temperature revealed multiple but unresolved chemical shifts of phenyl hydrogen atoms. The complicated structure of 'H-NMR of 6 comes from the presence of fluorine atoms or/and its other possible solution structures as illustrated in Figure 2. However, complication from fluorine atoms is excluded because a similar difluorinated derivative 5 exhibits two distinct types of phenyl stretches. 4a through $4 h$ were also characterized with spectroscopic methods.

\section{Conclusion}

$N$-sustituted- $\beta$-fluoropyrrole derivative, i.e., ethyl-4fluoro-1-methyl-5-phenylpyrrole and its higher congener were successfully synthesized by one of the following three reaction conditions: the reaction of $\mathbf{5}$ with acetic anhydride and triethylamine, the reaction of 6 with triethylamine, and the spontaneous conversion reaction of 5 . A possible mechanism is proposed based on the isolation and identification of the reaction intermediates $(5,6)$. The isolated intermediates support the presence of an acid proton, which would permit the facile attack of nucleophiles such as amines on the difluoropyrrole derivatives. Establishment of an anionic reaction pathway, including disubstituted $\beta$-fluoropyroles, would allow high yields of selective $\beta$-fluoropyrrole derivatives. Thereby, the mono $\beta$-fluoropyrroles would be useful to design systems of biological importance, such as porphyrins and branched pyrrole amides.

Acknowledgements. This study was funded by Wonkwang University (2005).

\section{References}

I. (a) Welch, J. T. Tetrahedron Lett. 1987, 43, 3123. (b) Marquez, V. E.; Tseng, C. K.; Mitsuya, H. J. Med. Chem. 1990, 33, 978.

2. (a) Yamazaki, T.; Welch, J. T.; Plummer, J. S.; Gimi, R. Tetrahedron Lett. 1991, 32, 4267. (b) Davis, J. A.; Han, W. Tetrahedron Lett. 1992, 33, 1153 . (c) Maqbool, A. S.; Victor, E, M. Trahedron Lett. 1994, 35, 3263.

3. Bass Jt., J. B.; Farer, L. S.; Hopewell, P. C.; O'Brian, R.; Jacobs, R. F.; Ruben, F.; Snider Jr, D. E.; Thomton, G. An. J. Respir. Crit. Care Med. 1994, 149, 1359.

4. Davidson, P. T.; Le, H. Q. Diugs 1992, 43, 651.

5. Grosset, J. H. Bull. Int. Union Tuberc. Lung Dis. 1990, 65, 86.

6. (a) Fried, J.; Hallinan, E. A. J. Am Chem. Soc. 1984, 106, 387 I. (b) Thaisrivongs, S.; Pals, W. H.; Thomasco, L. M. J. Med. Chen. 1985, 28, 1555. (c) Filler, R. Chent. Tech. 1974, 4, 752.

7. Buhr, G. Chem. Ber 1973, 106, 3544.

8. Leroy, J.; Rubinstein, M.; Wakselman, C. J. Fluorine. Chem. $1984,25,255$.

9. Onda, H.; Toi, H.; Aoyama, Y.; Ogoshi, H. Tetrahedron Lett. $1985,26,4221$.

10. Barmes, K. D.; Hu, Y.; Hunt, D. A. Synthetic Communications $1994,24,1749$.

11. Qui, Z. M.; Burton, D. J. Tetrahedron Lett, 1994, 35, 1813.

12. Qui, Z. M.; Burton, D. J. Tetrahedron Lett, 1994, 35,4319.

13. Boyd, G. V.; Wright, J. C. S. Perkin I 1972, 909.

14. Lee, Y.; Park, K.; Cho, I.; Chai, K. Journal of the Korean Chentical Society 1998, 42(3), 335-339.

15. Leroy, J.; Porhiel, E.; Bondon, A. Tetrahedron 2002, 58, 6713-6722. 16. Killean, R. C. G; Lawrence, J. L. Acta Cryst. Sect B 1969, 25, 1750. 